\title{
PARTICIPAÇÃO FEMININA NA FORÇA DE TRABALHO METROPOLITANO: O PAPEL DO STATUS SOCIOECONÔMICO DAS FAMÍLIAS
}

\author{
Lauro Ramos * \\ Marina Ferreira Fortes Aguas ${ }^{\dagger}$ \\ Luana Moreira de Souza Furtado ${ }^{\ddagger}$
}

\begin{abstract}
Resumo
Este trabalho analisa o comportamento das mulheres cônjuges como participantes no mercado de trabalho metropolitano. Procurou-se mostrar que o entorno familiar em que a mulher se encontra influi sobre a sua decisão de fazer parte da força de trabalho e também afeta a importância relativa dos determinantes tradicionais. As evidências levantadas indicam que, de fato, famílias consideradas potencialmente pobres têm uma probabilidade mais alta de ter a mulher na força de trabalho. Esse diferencial, contudo, é substancialmente reduzido, e mesmo revertido, quando elas possuem filhos em idade pré-escolar. Daí a importância de política públicas que facilitem o acesso dessas mulheres ao mercado de trabalho, como a oferta de creches, de modo a possibilitar o resgate de suas famílias da pobreza.
\end{abstract}

Palavras-chave: Mercado de trabalho, Participação Feminina, Determinantes do Ingresso.

\begin{abstract}
This study has analyzed the participation of married women in Brazilian metropolitan labor force. Specifically, we tried to show that the family environment in which the woman is has influence on their decision to join the workforce and affects the relative importance of traditional determinants. The evidence raised indicates that handicapped families have a higher probability to have women in the workforce, but the gap is substantially reduced, and even reversed, if they have children of preschool age. Thus the implementation of public policies that make it easier for these women to join the labor force, such as access to childcare, will prove useful to help to rescue their families from poverty.
\end{abstract}

Keywords: Female Participation, Determinants of Women Participation, Labor Market.

JEL classification: J21,J16

\footnotetext{
* Técnico de Planejamento e Pesquisa da Diretoria de estudos Sociais do IPEA: lauro.ramos@ipea.gov.br.

† CEDE/UFF e Tecnologista em Informações Geográficas e Estatísticas do IBGE: mariffaguas@gmail.com

‡ Bolsista do Programa Nacional de Pesquisa em Desenvolvimento (PNPD) no IPEA: luana.furtado@ipea.gov.br.
} 


\section{Introdução}

Nas últimas décadas, a transformação do papel da mulher na economia e na sociedade como um todo acarretou uma elevação da participação feminina no mercado de trabalho em vários países, inclusive no Brasil. Tal fenômeno acabou por despertar o interesse na busca de uma melhor compreensão sobre o comprometimento da mulher com a força de trabalho. Constatou-se que, além da influência de fatores socioculturais, e até mesmo daqueles de ordem religiosa, o nível de desenvolvimento econômico e a natureza do funcionamento do mercado afetam esse envolvimento, que tem como principal indicador o nível de participação feminino. ${ }^{1}$

De modo geral, apesar da evolução positiva no passado recente, ainda parece haver um baixo aproveitamento da capacidade laboral das mulheres no mercado de trabalho brasileiro, na medida em que as taxas femininas são bem menores que as observadas para os países industrializados, bem como ficam abaixo das reportadas para os homens. Caso isso ocorra devido à existência de barreiras que dificultem a concretização do interesse das mulheres em ingressar na força de trabalho, a decorrente subutilização de recursos deve ser encarada como um empecilho para a redução da pobreza. Essas implicações podem ser ainda mais intensas se as barreiras ao ingresso das mulheres forem diferenciadas, de tal sorte que mulheres pertencentes a famílias em condições socioeconômicas mais adversas enfrentem dificuldades maiores no acesso ao mercado de trabalho.

De fato, a literatura empírica brasileira mostra que a participação feminina no mercado de trabalho é um elemento importante para retirar as famílias da condição de pobreza e que essa taxa sobe continuamente ao longo dos estratos de renda familiar per capita. A questão a ser averiguada, então, é por que razões as mulheres de famílias mais pobres têm um menos engajamento no mercado de trabalho.

Nesse contexto, o artigo procura contribuir para o melhor entendimento da questão do ingresso de mulheres por meio da avaliação de como características pessoais e domiciliares interferem na decisão de participar da força de trabalho. Mais importante, o estudo averígua a existência de diferenças nesses determinantes de acordo com o status socioeconômico da família a priori das decisões de ingresso no mercado de ambos os cônjuges, contrastando a sua importância no grupo em condições mais desfavoráveis vis-à-vis o contingente em melhor situação.

Além dessa introdução, o artigo contém, na seção seguinte, uma breve resenha de trabalhos sobre participação feminina no Brasil, seguida de uma seção com a descrição da evolução recente da presença das mulheres no mercado de trabalho. Na seção 4 são apresentadas a metodologia e critérios operacionais utilizados, e na próxima os resultados das estimações. Por fim, a seção 6 conclui o trabalho com um apanhado geral, destacando as principais conclusões.

\footnotetext{
${ }^{1}$ Durand (1975), por exemplo, sugere que o padrão de participação feminino no mercado de trabalho possui alto grau de vulnerabilidade a mudanças na demanda por mão de obra, variações nos níveis de renda e salários e taxas de desemprego, entre outros fatores extra-econômicos.
} 


\section{Resenha da Literatura no Brasil}

A participação feminina no mercado de trabalho tem sido alvo de vários estudos no Brasil, que constituem um grande esforço na tentativa de entender o nível e a evolução do comportamento dessa variável, assim como os determinantes do ingresso feminino no mercado de trabalho. Trabalhos como os de Melo (1982) e Camargo \& Serrano (1983) buscam no diferencial de salários entre homens e mulheres a causa da baixa taxa de participação feminina, concluindo que existe uma segregação por sexo contra a atuação das mulheres na força de trabalho. Barros et al. (1995) por outro lado, refutam a existência de segregação e apontam a discriminação por parte de empregadores como a justificativa para a dificuldade de inserção das mulheres no mercado.

Sedlacek \& Santos (1991), utilizam dados da PNAD de 1984 com a finalidade de analisar a relação entre a participação das mulheres cônjuges e a renda do marido, controlando pela influência de outras variáveis na propensão de oferta de trabalho dos cônjuges femininos, que guardam relação com o seu salário de reserva. A conclusão alcançada pelos autores estabelece uma relação negativa entre a taxa de participação feminina e a renda do marido, nos moldes mais simplistas do modelo renda x lazer. Ramos \& Soares (1995) mostram que ex post a decisão de ingresso da mulher, a relação entre taxa de participação e renda familiar per capita é positiva, destacando a contribuição da participação feminina para a redução da incidência de pobreza. A importância da estrutura familiar e do engajamento dos membros da família, homens e mulheres, no mercado de trabalho para diminuir a pobreza também é investigada em Fernandes et al. (2002), que encontram respaldo empírico para efeitos consideráveis, principalmente quando da redução do desemprego.

Quanto ao efeito da participação feminina sobre a desigualdade de rendimentos, Barros \& Mendonça (1989) mostram que do ponto de vista teórico o resultado não é simples de ser determinado e elencam algumas condições para que o impacto seja no sentido de redução. Os autores concluem que os rendimentos dos cônjuges femininos na época tinham "um efeito insignificante sobre a distribuição de renda entre as famílias." Analisando o período 1982-1997 com base simulações contrafactuais em relação às características observáveis e respectivos retornos, Scorzafave \& Menezes-Filho (2005) concluem que os efeitos do aumento da taxa de participação sobre a desigualdade são ambíguos, dependendo do índice de concentração utilizado. Analisando a questão sob um prisma diferente, por meio da decomposição do índice de Gini segundo os componentes da renda, Hoffmann \& Leone (2004) mostram que, dado o crescimento da importância dos rendimentos do trabalho da mulher na família, há um aumento da sua contribuição para a desigualdade da renda domiciliar per capita.

Em um trabalho recente, Costa (2007) retoma a estimação dos determinantes do ingresso das mulheres no mercado de trabalho e faz uma comparação entre da participação das mulheres mais pobres e das mais ricas. Embora os resultados sejam severamente comprometidos por problemas de seletividade e endogeneidade, a autora destaca que algumas variáveis que influenciam o salário de reserva e contribuem para diminuir a participação das mulheres, atuando de forma mais intensa nos estratos inferiores. Esse resultado está em linha com aquele destacado em Ramos \& Soares (1995), após a decisão de ingresso da mulher, não permitindo, portanto, estabelecer uma relação de causalidade. 
A contribuição almejada no âmbito desse trabalho é a pesquisa de elementos úteis para a compreensão de uma possível relação a priori entre a probabilidade de participar do mercado de trabalho e o status socioeconômico da família. Ou seja, investigar os determinantes da participação das mulheres em famílias em condições socioeconômicas mais adversas - e que têm uma probabilidade mais elevada de serem pobres que as outras -, cotejando-os com os mesmos determinantes em famílias em condições mais favoráveis. Além de evitar os entraves econométricos criados pelos problemas mencionados acima, a busca de uma relação apriorística tem a vantagem de esta estar relacionada com fatores de cunho mais estrutural, que plausivelmente refletem melhor o conceito de renda permanente, do que por meio da renda corrente das famílias.

\section{A Participação das Mulheres no Mercado de Trabalho Brasileiro no Passado Recente}

A trajetória da taxa de atividade feminina no Brasil, com idade revela um aumento da participação das mulheres de 15 e 64 anos idade ${ }^{2}$ entre 2001 e 2005, quando passou de $58 \%$ para $63 \%$, permanecendo estável daí em diante. Já homens mantiveram sua participação praticamente no mesmo patamar nesse período, em torno de $85 \%,{ }^{3}$ de modo que ainda persiste uma diferença na participação por gênero da ordem de 22 p.p em 2008.

A figura 1 exibe a evolução na década da taxa de atividade feminina em alguns países. ${ }^{4}$ Entre 2001 e 2008, todos os países analisados apresentaram uma trajetória de ascensão ou estabilidade em suas taxas de participação. Entretanto, é possível observar como o nível dessas taxas variou entre eles. Os Estados Unidos e a Austrália, nos dois últimos anos, superaram 70\% de mulheres entre 15 e 64 anos na força de trabalho. O Brasil acompanhou o crescimento da Europa dos 15 e do Japão até 2005, depois se estabilizou enquanto os demais continuaram a subir, de tal forma que, em 2008, as diferenças se tornam praticamente as mesmas, ou até maiores, que aquelas observadas no início da década. Por fim, o México, apesar de uma elevação de mais de 6 p.p. na taxa de atividade feminina, foi o país com o menor nível de participação em todo período dentre aqueles mostrados aqui.

Para os fins desse estudo é necessário investigar, no universo das regiões metropolitanas brasileiras, a distribuição das mulheres no mercado de trabalho segundo sua desagregação por posição no domicílio - chefes ${ }^{5}$, cônjuges, filhas, parentes e outras - de forma a verificar a representatividade daquelas que são consideradas cônjuges. A tabela 1 mostra essa composição para as mulheres na população economicamente ativa (PEA) metropolitana, revelando que os cônjuges femininos formam, por larga margem, o grupo com maior participação na força de trabalho, tendo crescido nos últimos anos e atingido

\footnotetext{
${ }^{2}$ Esse intervalo de idade foi escolhido de forma a tornar os dados brasileiros comparáveis aos da OCDE.

${ }^{3}$ Esses valores foram obtidos por elaboração própria a partir das PNADs e estão disponíveis mediante solicitação.

${ }^{4} \mathrm{O}$ site da OECD apresenta essa informação para todos os seus membros.

${ }^{5}$ Para melhor adequação aos propósitos do estudo foi redefinido o conceito de chefe de domicílio utilizado pelo IBGE, que passou a ser restrito às mulheres sem companheiros, sendo as demais, que se declaram chefes na PNAD, mas que possuem um companheiro, englobadas no grupo dos cônjuges.
} 
$47,4 \%$ em 2008. Cabe atentar também para a proporção de mulheres chefes que cresceu entre 2001 e 2008 , cerca de 2 p.p., tornando-se praticamente $20 \%$ da PEA feminina em 2008.

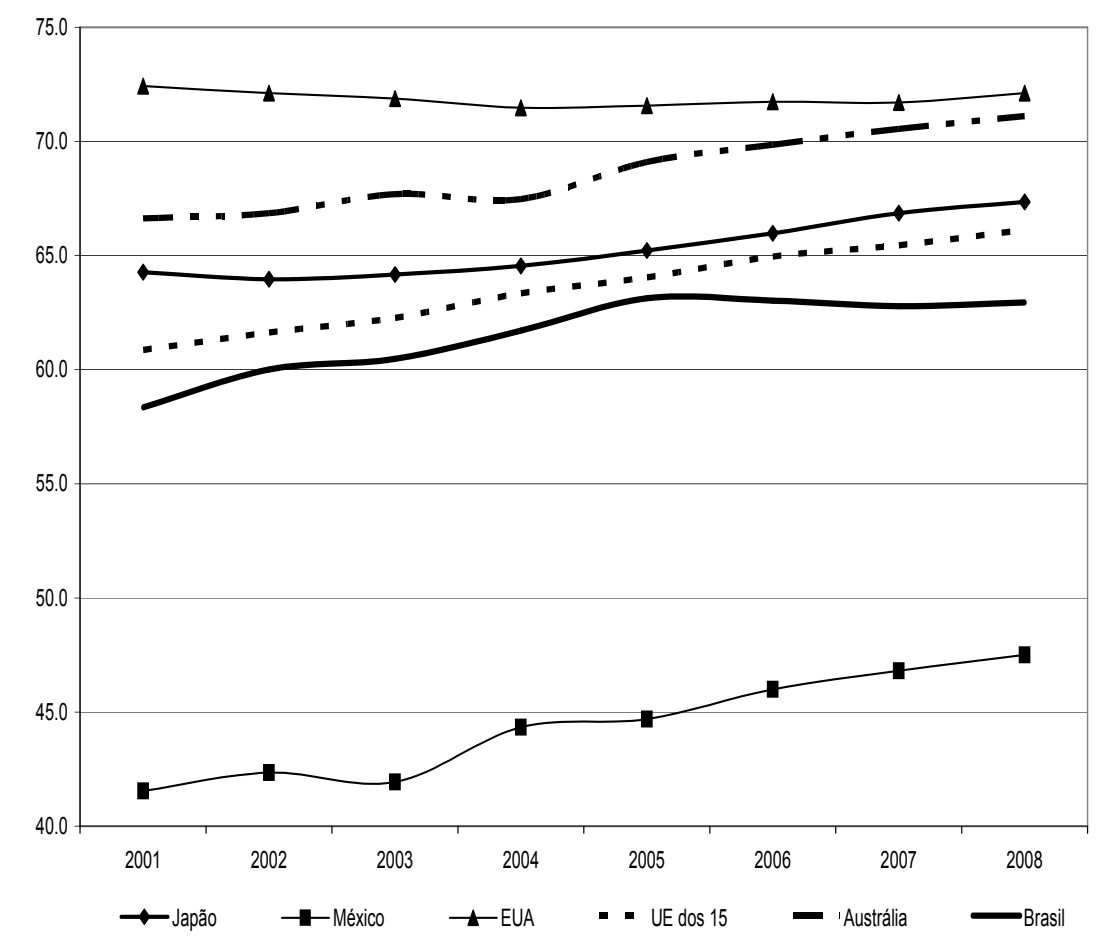

Fonte: Elaboração própria a partir dos dados da OCDE e da PNAD

Figura 1: Taxa de participação das mulheres entre 15 e 64 anos - Vários Países

Tabela 1: Mulheres na PEA entre 15 e 64 anos de idade por condição no domicílio - Brasil Metropolitano

\begin{tabular}{lccccc}
\hline Ano & Chefe & Cônjuge & Filha & Parente & Outros \\
\hline 2001 & 18,0 & 45,7 & 27,2 & 6,8 & 2,4 \\
2002 & 18,1 & 45,8 & 27,4 & 6,5 & 2,2 \\
2003 & 18,9 & 46,0 & 27,0 & 6,2 & 1,9 \\
2004 & 18,8 & 45,8 & 27,3 & 6,4 & 1,7 \\
2005 & 18,6 & 46,6 & 27,3 & 6,0 & 1,5 \\
2006 & 19,2 & 46,3 & 26,4 & 6,5 & 1,6 \\
2007 & 19,2 & 46,5 & 26,1 & 6,5 & 1,6 \\
2008 & 19,9 & 47,4 & 25,2 & 5,9 & 1,6 \\
\hline Fonte: \\
Elaboração própria a partir da PNAD.
\end{tabular}

Logo, a participação feminina brasileira apresentou uma evolução positiva tanto no cenário internacional quanto internamente. Persiste, todavia, um grande diferencial entre as taxas de participação segundo gênero que pode 
estar sinalizando que ainda existe uma subutilização laboral das mulheres. Adicionalmente, quando o universo de análise é Brasil metropolitano, as mulheres cônjuges já representam quase metade da força de trabalho feminina, enquanto as chefes equivalem a $1 / 5$ da PEA.

\section{Marco teórico e estratégia empírica}

O marco teórico mais tradicional para a análise da alocação do tempo da mulher é baseado em um modelo teórico proposto por Becker (1965) que permite investigar a natureza do processo de decisão no contexto familiar. O tempo do casal usualmente é entendido como sendo dividido entre: a) atividades de mercado, dado pelas suas atividades produtivas fora da esfera domiciliar; b) produção doméstica, associada às atividades ligadas ao domicílio (como limpeza e reparos, entre outros), produção de commodities (como o preparo de refeições) e à família (educação dos filhos, por exemplo); e c) lazer estrito senso. A maximização da utilidade familiar fornece a quantidade de horas que cada um dos cônjuges dedica ao trabalho, à produção doméstica e ao lazer. Em muitas aplicações, este modelo é simplificado pelo agrupamento de produção doméstica e lazer propriamente dito sob a rubrica "lazer".

Adotando essa simplificação, podemos considerar que a família toma suas decisões de modo a tentar maximizar sua função utilidade conjunta, sendo $U=U_{F}\left(x, L_{h}, L_{m}\right)$, onde $U_{F}(\cdot)$ é a função utilidade da família, $x$ é a cesta de bens adquiridos no mercado. $L_{h}$ é a quantidade de "lazer" do cônjuge masculino e $L_{m}$ a quantidade de "lazer" do cônjuge feminino, sujeito a uma restrição orçamentária e uma restrição de tempo. ${ }^{6}$

Existem três tipos distintos de rendas no modelo: renda não-trabalho, renda do trabalho do cônjuge masculino e renda do trabalho do cônjuge feminino, que serão representadas por: $\bar{Y}^{f}, W_{h} H_{h}, W_{m} H_{m}$ respectivamente, sendo $W_{i}$ o salário-hora recebido pelo indivíduo $i$ e $H_{i}$ as horas por ele trabalhadas $(i=h$ (homem), $m$ (mulher)). O gasto total com o consumo da cesta de bens adquirido no mercado é dado por seus preços $(p)$ e pelas respectivas quantidades $(x)$ Nestes termos, a restrição orçamentária da família é:

$$
p x=\bar{Y}^{f}+W_{h} H_{h}+W_{m} H_{m} .
$$

A família pode dividir o tempo disponível de seus membros $(T)$, por simplicidade admitido igual para ambos, entre o tempo dedicado às atividades de mercado $(H)$, e o tempo dedicado tanto para o "lazer" $(L)$. A restrição de tempo da família é:

$$
H_{h}+L_{h}=T ; H_{m}+L_{m}=T .
$$

O problema de decisão familiar de oferta de trabalho pode, então, ser formalizado da seguinte forma:

$$
\underset{L_{h}, L_{m}, x}{\operatorname{Max}} U=U_{F}\left(x, L_{h}, L_{m}\right),
$$

\footnotetext{
${ }^{6}$ Muitas vezes, por simplicidade, adota-se o modelo sequencial. Na sua versão mais simples, o chamado modelo "chauvinista", há apenas dois estágios decisórios: no primeiro, o marido otimiza sua oferta pressupondo que a mulher não trabalhará, e, no segundo, ela otimiza sua oferta tomando como dada a decisão do primeiro.
} 
sujeito a: $p x+W_{h} L_{h}+W_{m} L_{m}=\bar{Y}^{f}+\left(W_{h}+W_{m}\right) T$

A solução ótima $\left(x^{*}, L_{h}^{*}, L_{m}^{*}\right)$ implica a oferta de trabalho feminina $H_{m}^{*}=$ $T-L_{m}^{*}$.

As principais implicações, no caso de solução interior e admitindo que $x$ e $L$ são bens normais, para a quantidade de tempo alocado pela mulher no mercado de trabalho são que a sua oferta de trabalho tende a diminuir com o crescimento da renda não-trabalho da família e com seu salário de mercado, e a diminuir com o salário do cônjuge.

A situação interessante para a questão do ingresso é a possibilidade de soluções de canto $-L_{m}^{*}=T, H_{m}^{*}=0$ - que é tão mais provável quanto mais baixo for o salário de mercado para a mulher. O menor salário de mercado que faz com que a mulher ingresse na força de trabalho é dito salário de reserva e varia inversamente com sua renda não-trabalho. Como lazer está associado à produção doméstica, a taxa marginal de substituição entre tempo devotado ao mercado e ao lazer está associado à produtividade do tempo da mulher na produção doméstica e, portanto, ao seu salário de reserva também.

É possível, a princípio, modelar a probabilidade de participação da mulher através de: ${ }^{7}$

$$
P_{i}=f\left(X_{i}, Z_{i}, \bar{Y}_{i}^{m}\right)
$$

onde,

$P_{i}$ : probabilidade de participação do cônjuge feminino i;

$X_{i}$ : atributos produtivos do cônjuge feminino i;

$Z_{i}$ : características da estrutura familiar do i-ésimo cônjuge feminino;

$\bar{Y}_{i}^{m}$ : renda não-trabalho do cônjuge feminino, cuja derivada parcial é negativa na medida em que seu lazer for um bem normal.

Como o salário de reserva é uma variável não observada que está associada aos atributos produtivos da mulher e às características da estrutura familiar em que ela está inserida, o que se observa é apenas a sua decisão de ingresso ou não, que depende de uma variável não observável, ou latente. Nesse caso, um modelo apropriado para a participação da mulher na força de trabalho é um probit, onde a variável dependente é dicotômica: participa da força de trabalho $(\mathrm{Y}=1)$ ou não participa da força de trabalho $(\mathrm{Y}=0)$. Sua forma funcional na estimativa destes parâmetros é:

$$
\operatorname{Prob}(Y=1)=\Phi\left(\mathbf{X} \beta+\mathbf{Z} \gamma+\overline{\mathbf{Y}}_{\mathbf{i}}^{\mathbf{m}} \delta\right),
$$
de: ${ }^{8}$

onde $\Phi$ é a função acumulada da normal padrão e, resultando na estimação

$$
Y_{i}^{\prime}=X_{i} \beta+Z_{i} \gamma+\delta \bar{Y}_{i}^{m}{ }_{i}+u_{i}
$$

onde $u_{i}$ é um termo aleatório com distribuição $N:\left(0, \sigma^{2}\right)$ e $Y_{i}^{\prime}$ é uma variável latente tal que $Y_{i}=1$ se $Y_{i}^{\prime}>0$ e $Y_{i}=0$ caso contrário.

Os parâmetros $\beta, \gamma$ e $\delta$ são estimados por máxima verossimilhança e representam o impacto das variáveis independentes sobre a variável latente e não sobre a probabilidade propriamente dita. O impacto sobre essa última é

\footnotetext{
${ }^{7} \mathrm{Na}$ verdade essa é a forma reduzida. A forma estrutural contém explicitamente o salário de reserva. Além disso, o modelo em que está baseada é o "chauvinista".

${ }^{8} \mathrm{Ou} Y_{i}=\Phi\left(X \beta+Z \gamma+\bar{Y}_{i}^{m} \delta\right)+\varepsilon_{i}$
} 
dito efeito marginal e corresponde à derivada parcial da variável latente em relação á variável em questão, no mais das vezes avaliada no valor médio das variáveis independentes.

As variáveis independentes utilizadas são a educação e idade do homem ${ }^{9}$ e da mulher, ${ }^{10}$ a região metropolitana de residência, a presença de filhos entre 0 e 5, 6 e 10 e, 11 e 17 anos de idade. Além disso, será utilizado um indicador de situação socioeconômica das famílias, apresentado a seguir, e sua interação com a presença de filhos menores.

\subsection{Indicador de Status Socioeconômico}

Para verificar a existência de diferenças de efeitos nos determinantes do ingresso das mulheres no mercado de trabalho em situação de maior propensão à pobreza ou não da família, e estabelecer relações de causalidade subjacentes, lançaremos mão de um indicador do status socioeconômico da família anterior às decisões de ingresso de ambos os cônjuges, privilegiando características mais permanentes e, portanto, uma noção mais estrutural da pobreza. Para tanto foi incluída na especificação a variável $D$, definida pela partição segundo as seguintes dummies:

$$
\begin{aligned}
& D^{-}=1 \text { se } \bar{Y}^{f}=0, S_{h} \leq 8 \text { e } S_{m} \leq 8 ; D^{-}=0 \text { caso contrário; } \\
& D^{+}=1 \text { se } \bar{Y}^{f}>0 \text { e }\left(S_{h} \geq 11 \text { ou } S_{m} \geq 11\right) ; D^{+}=0 \text { caso contrário; } \\
& D^{0}=1 \text { se } D^{-}=D^{+}=0 ; D^{0}=0 \text { caso contrário; }
\end{aligned}
$$

onde $\bar{Y}^{f}$ é a renda não-trabalho da família e $S_{h}, S_{m}$ representam o número de anos completos de estudo dos cônjuges masculino e feminino.

Assim, as famílias integrantes do grupo $D^{-}$são aquelas sem renda nãotrabalho e onde nenhum dos cônjuges foi além de completar o ensino fundamental. Já aquelas pertencentes ao grupo $D^{+}$possuem alguma fonte de renda não-trabalho e têm pelo menos um dos cônjuges com ensino médio completo. As restantes formam o grupo chamado aqui de "referência". ${ }^{11}$

A motivação para adotar esse indicador é que ele seja uma boa proxy do situação socioeconômico, ou potencial de renda da família pré-mercado de trabalho. Assim, por meio da combinação da escolaridade dos cônjuges e da renda não-trabalho da família são identificadas aquelas com propensão maior de pertencer aos estratos mais pobres $\left(D^{-}\right)$ou de se situar nos estratos mais elevados $\left(D^{+}\right)$da população pós-decisão de ingresso, tornando possível captar os efeitos do status socioeconômico sobre o salário de reserva da mulher. ${ }^{12}$

\footnotetext{
${ }^{9}$ Que servem para captar os efeitos dos seus rendimentos.

${ }^{10}$ Para as mulheres na forma de categorias (faixas etárias de 15-19, 20-29, 30-39, 40-49 e 50-60 anos de idade e ensino fundamental incompleto, do fundamental completo ao médio incompleto, do médio completo ao superior incompleto e acima de superior completo para os grupos de escolaridade), enquanto para os homens de forma contínua.

${ }^{11}$ A título de ilustração, vale reportar (ver Anexo A.1) que em 2008 o primeiro grupo continha quase $22 \%$ das famílias e o segundo pouco mais de $10 \%$ delas.

${ }^{12}$ De fato, conforme pode ser visto no Apêndice A.1, a distribuição para o ano de 2008 desse indicador segundo os décimos da distribuição ex post da renda familiar per capita revela que 59,6\% das mulheres de $D^{-}$encontravam-se nos quatro primeiros décimos e $8,1 \%$ nos três últimos; para $D^{+}, 45,8 \%$ delas encontravam-se nos três décimos do topo e $30,7 \%$ nos quatro debaixo, enquanto o grupo de referência apresentava uma distribuição praticamente uniforme $(33,5 \%$ nos quatro primeiros décimos, $31,1 \%$ nos três intermediários e 35,4\% nos três décimos de cima).
} 


\subsection{Base de Dados}

A análise empírica desenvolvida no artigo utiliza os microdados da Pesquisa Nacional por Amostra de Domicílios (PNAD), elaborada pelo IBGE, de 2001 a 2008, centrada nesse último ano. A amostra selecionada é composta pelas mulheres entre 15 e 50 anos, residentes em domicílios particulares permanentes e unifamiliares das 10 regiões metropolitanas brasileiras, que na semana de referência faziam parte de famílias nucleares ${ }^{13}$. As famílias cujas mulheres não apresentavam um parceiro foram retiradas da amostra de modo a homogeneizar o entorno familiar, haja vista que a presença, ou não, do cônjuge têm um papel importante nas escolhas da mulher. Da mesma forma, a presença de outros membros que não os cônjuges também podem afetar essas escolhas. O tamanho final da amostra é de cerca de 20 mil observações, e as principais estatísticas descritivas são mostradas no anexo.

\section{Resultados}

Na tabela a seguir são apresentados os resultados dos três modelos estimados para a participação da mulher na força de trabalho no ano de 2008. Os valores reportados correspondem aos coeficientes de cada variável dependente sobre a probabilidade de ingresso da mulher e, entre parênteses, os respectivos erros robustos.

A primeira coluna contém os resultados da estimação de uma regressão padrão, que tem como variáveis independentes apenas aquelas que dizem respeito à estrutura da família, como presença de filhos e condição da mulher, e as que descrevem os seus atributos produtivos e de seu cônjuge. ${ }^{14}$ Em geral, as estimativas obtidas estão em consonância com as expectativas e com os resultados mais comuns na literatura. A presença de crianças em idade préescolar afeta de forma negativa a probabilidade de participação, a presença de filhos de 6 a 10 anos tem efeito estatisticamente significativo, porém de menor magnitude, enquanto filhos acima dessa idade parecem não interferir na decisão de ingresso na força de trabalho pelas mulheres. Além disso, o pico de probabilidade de participação feminina ocorre na faixa etária entre $30 \mathrm{e}$ 39 anos de idade, seguida pela faixa posterior, de 40 a 50 anos, refletindo em alguma medida o ciclo de fecundidade e casamento.

Com relação à escolaridade da mulher, anos completos de estudo elevam a sua probabilidade de fazer parte da força de trabalho como consequência da elevação de seu custo de oportunidade, uma vez que o salário de mercado cresce com a escolaridade, tanto para as mulheres quanto para os homens. No que tange à evolução da importância desses determinantes ao longo da década, mostrada no anexo, parece haver uma tendência de ligeira perda de importância da maioria deles, plausivelmente refletindo uma disseminação da decisão de ingressar em todos os grupos considerados.

As variáveis de escolaridade e experiência do homem, como proxy para seus rendimento, apresentam um sinal negativo. Isso condiz com a ideia de que a renda do companheiro tem um efeito de reduzir a participação da mulher. É bem possível que essa variável também capte elementos sócio-culturais

\footnotetext{
${ }^{13}$ Famílias nucleares são aquelas compostas pelo chefe de domicílio, seu cônjuge e filhos, quando presentes.

${ }^{14}$ Os resultados para as dummies associadas às regiões metropolitanas estão disponíveis com os autores.
} 
Tabela 2: Participação do cônjuge feminino na força de trabalho em 2008 - Coeficientes

\begin{tabular}{|c|c|c|c|}
\hline Tem filhos de 0 a 5 anos & $\begin{array}{c}-0,397^{* * *} \\
(0,028)\end{array}$ & $\begin{array}{c}-0,401^{* * *} \\
(0,029)\end{array}$ & \\
\hline Tem filhos de 6 a 10 anos & $\begin{array}{c}-0,130^{* * *} \\
(0,026)\end{array}$ & $\begin{array}{c}-0,120^{* * *} \\
(0,027)\end{array}$ & \\
\hline Tem filhos de 11 a 17 anos & $\begin{array}{l}0,024 \\
(0,027)\end{array}$ & $\begin{array}{l}0,028 \\
(0,033)\end{array}$ & $\begin{array}{l}0,028 \\
(0,034)\end{array}$ \\
\hline \multicolumn{4}{|l|}{ Conjuge entre 15 e 19 anos } \\
\hline Conjuge entre 20 e 29 anos & $\begin{array}{l}0,609^{* * *} \\
(0,091)\end{array}$ & $\begin{array}{l}0,607^{* * *} \\
(0,092)\end{array}$ & $\begin{array}{l}0,603^{* * *} \\
(0,092)\end{array}$ \\
\hline Conjuge entre 30 e 39 anos & $\begin{array}{l}0,671^{* * *} \\
(0,093)\end{array}$ & $\begin{array}{l}0,654^{* * *} \\
(0,094)\end{array}$ & $\begin{array}{l}0,646^{* * *} \\
(0,095)\end{array}$ \\
\hline Conjuge entre 40 e 50 anos & $\begin{array}{l}0,532^{* * *} \\
(0,097)\end{array}$ & $\begin{array}{l}0,514^{* * *} \\
(0,099)\end{array}$ & $\begin{array}{l}0,506^{* * *} \\
(0,099)\end{array}$ \\
\hline \multicolumn{4}{|l|}{ Escolaridade entre 0 e 7 anos } \\
\hline Escolaridade entre 8 e 11 anos & $\begin{array}{l}0,055 \\
(0,034)\end{array}$ & $\begin{array}{l}0,067^{*} \\
(0,035)\end{array}$ & $\begin{array}{l}0,070^{* *} \\
(0,035)\end{array}$ \\
\hline Escolaridade entre 11 anose superior incompleto & $\begin{array}{l}0,317^{* * *} \\
(0,033)\end{array}$ & $\begin{array}{l}0,368^{* * *} \\
(0,036)\end{array}$ & $\begin{array}{l}0,370^{* * *} \\
(0,036)\end{array}$ \\
\hline Escolaridade maior ou igualao superior completo & $\begin{array}{l}0,967^{* * *} \\
(0,054)\end{array}$ & $\begin{array}{l}1,019^{* * *} \\
(0,057)\end{array}$ & $\begin{array}{l}1,028^{* * *} \\
(0,057)\end{array}$ \\
\hline Experiência do homem & $\begin{array}{c}-0,013^{* * *} \\
(0,002)\end{array}$ & $\begin{array}{c}-0,012^{* * *} \\
(0,002)\end{array}$ & $\begin{array}{c}-0,012^{* * *} \\
(0,002)\end{array}$ \\
\hline Escolaridade do homem & $\begin{array}{c}-0,022^{* * *} \\
(0,004)\end{array}$ & $\begin{array}{c}-0,016^{* * *} \\
(0,004)\end{array}$ & $\begin{array}{c}-0,016^{* * *} \\
(0,004)\end{array}$ \\
\hline$D^{-}$ & & $\begin{array}{l}0,067^{*} \\
(0,036)\end{array}$ & $\begin{array}{l}0,103^{* *} \\
(0,047)\end{array}$ \\
\hline$D^{+}$ & & $\begin{array}{c}-0,186^{* * *} \\
(0,042)\end{array}$ & $\begin{array}{c}-0,293^{* * *} \\
(0,056)\end{array}$ \\
\hline Interlação entre ter filhosentre 0 e 5 anos e $D^{-}$ & & & $\begin{array}{c}-0,474^{* * *} \\
(0,057)\end{array}$ \\
\hline Interlação entre ter filhosentre 0 e 5 anos e Dref & & & $\begin{array}{c}-0,398^{* * *} \\
(0,034)\end{array}$ \\
\hline Interlação entre ter filhosentre 0 e 5 anos e $D^{+}$ & & & $\begin{array}{c}-0,254^{* * *} \\
(0,088)\end{array}$ \\
\hline Interlação entre ter filhosentre 6 e 10 anos e $D^{-}$ & & & $\begin{array}{c}-0,165^{* * *} \\
(0,055)\end{array}$ \\
\hline Interlação entre ter filhosentre 6 e 10 anos e Dref & & & $\begin{array}{c}-0,132^{* * *} \\
(0,033)\end{array}$ \\
\hline Interlação entre ter filhosentre 6 e 10 anos e $D^{+}$ & & & $\begin{array}{l}0,058 \\
(0,080)\end{array}$ \\
\hline Observações $^{2}$ & 17888 & 17394 & 17394 \\
\hline
\end{tabular}

Fonte: As regressões são estimadas usando um modelo Probit. Os valores reportados se referem aos coeficientes. Os erros-padrão robustos são reportados entre parênteses. A amostra é composta por mulheres entre 15 e 50 anos que possuem um * siginicativo a $10 \%{ }^{* *}$ significativo a $5 \%$; ${ }^{* * *}$ significativo a $1 \%$ Nota 1 : O universo de análise considera apenas as famílias nucleares. Nota 2: Em todos os modelos foram utilizadas dummies para as regiões metropolitanas. Fonte: PNAD entre 2001 e 2008 do IBGE. 
da natureza da formação dos pares conjugais, mas a associação com a renda do cônjuge masculino prevalece.

A influência do status socioeconômico fica patente nos resultados do modelo da segunda coluna, que passa a incluir o indicador D. Em 2008, uma mulher pertencente ao grupo mais carente em termos de condições socioeconômicas $\left(D^{-}\right)$tinha uma probabilidade maior de participar do mercado do que a da categoria de referência, o contrário ocorrendo para o grupo das famílias em condições mais favoráveis $\left(D^{+}\right)$, refletindo as diferenças nas taxas de substituição marginal entre bens de mercado e produção doméstica entre esses contingentes.

Por fim, o modelo da terceira coluna inclui a interação entre o indicador $D$ e as variáveis relacionadas à presença de filhos em idade pré-escolar $(0$ a 5 anos de idade) e aqueles com idade para cursar os primeiros anos do ensino fundamental (6 a 10 anos), de forma a avaliar possíveis efeitos adicionais no status socioeconômico pré-mercado de trabalho da família sobre a participação feminina nesse mercado. Em que pese a presença de filhos menores ao contribuir para a diminuição da participação no mercado, em consonância com os resultados dos modelos anteriores, esse efeito é claramente maior para o grupo de mulheres em famílias com maior probabilidade de serem pobres do que para aquelas potencialmente mais ricas ${ }^{15}$. Além disso, os coeficientes são bastante significativos para os três grupos do indicador $D$ nas duas faixas etárias de filhos, exceto na interação entre $D^{+}$e a presença de filhos de 6 a 10 anos de idade. Esses resultados confirmam a tese de que o status socioeconômico afetam a participação feminina no mercado.

A próxima tabela apresenta os efeitos marginais de cada variável do terceiro modelo na probabilidade de participação da mulher para os anos de 2001, 2005 e 2008, que são calculados, como de hábito, nos valores médios das variáveis, e na variação de 0 para 1 no caso das dummies. Assim, a interpretação da intensidade do efeito de cada variável fica mais simples, ressalvado o fato de que ele varia ao longo da distribuição.

O efeito marginal associado ao indicador de condições socioeconômicas contribui para aumentar a probabilidade de participação das mulheres em famílias do menos aquinhoadas em pouco mais de 6 p.p. em 2001 e 2005, caindo para 3,6 p.p. em 2008. Para as mulheres em famílias do outro extremo há, entretanto, uma redução por volta de 10 p.p.

A interação do entorno familiar com a presença de filhos em idade préescolar tem efeitos marginais expressivos, principalmente para o grupo $D^{-}$: $-21,4$ p.p. em $2001,-16,4$ p.p. em 2005 e -18,0 p.p. em 2008. Nos outros dois grupos, esses efeitos são de magnitude menor, porém ainda consideráveis, sendo cerca de metade desses valores para o caso de $D^{+}$. Já no caso da interação desse indicador com a presença de filhos de 6 a 10 anos, a intensidade do efeito marginal é bem menor, atingindo um pico de $-6,0$ p.p. para as mulheres em famílias potencialmente pobres em 2008 e sendo inexistente para aquelas potencialmente ricas. Vale ressalvar que, diferentemente do que ocorreu no caso da presença de crianças de até 5 anos de idade, ele cresceu ao longo da década no âmbito do grupo $D^{-}$.

\footnotetext{
${ }^{15} \mathrm{O}$ teste com a hipótese nula de que esses dois coeficientes são iguais no caso da presença de filhos em idade pré-escolar tem um p-valor de 0,023 e para filhos de 6 a 10 anos de idade ele é igual a 0,021 .
} 
Tabela 3: Participação do cônjuge feminino na força de trabalho em 2001, 2005 e 2008 - Efeitos Marginais

\begin{tabular}{|c|c|c|c|}
\hline & \multicolumn{3}{|c|}{ Ano } \\
\hline & 2001 & 2005 & 2008 \\
\hline Tem filhos de 11 a 17 anos & $\begin{array}{c}0,017 \\
(0,011)\end{array}$ & $\begin{array}{l}0,022^{* *} \\
(0,010)\end{array}$ & $\begin{array}{l}0,012 \\
(0,010)\end{array}$ \\
\hline Conjuge entre 15 e 19 anos & & & \\
\hline Conjuge entre 20 e 29 anos & $\begin{array}{l}0,196^{* * *} \\
(0,027)\end{array}$ & $\begin{array}{l}0,144^{* * *} \\
(0,026)\end{array}$ & $\begin{array}{l}0,196^{* * *} \\
(0,026)\end{array}$ \\
\hline Conjuge entre 30 e 39 anos & $\begin{array}{l}0,250^{* * *} \\
(0,028)\end{array}$ & $\begin{array}{l}0,168^{* * *} \\
(0,028)\end{array}$ & $\begin{array}{l}0,218^{* * *} \\
(0,030)\end{array}$ \\
\hline Conjugue entre 40 e 50 anos & $\begin{array}{l}0,169^{* * *} \\
(0,031)\end{array}$ & $\begin{array}{l}0,112^{* * *} \\
(0,030)\end{array}$ & $\begin{array}{l}0,171^{* * *} \\
(0,032)\end{array}$ \\
\hline Escolaridade entre 0 e 7 anos & & & \\
\hline Escolaridade entre 8 e 11 anos & $\begin{array}{l}0,047^{* * *} \\
(0,013)\end{array}$ & $\begin{array}{l}0,074^{* * *} \\
(0,011)\end{array}$ & $\begin{array}{l}0,024^{* *} \\
(0,012)\end{array}$ \\
\hline Escolaridade entre 11 anos e superior incompleto & $\begin{array}{l}0,186^{* * *} \\
(0,014)\end{array}$ & $\begin{array}{l}0,169^{* * *} \\
(0,012)\end{array}$ & $\begin{array}{l}0,127^{* * *} \\
(0,012)\end{array}$ \\
\hline Escolaridade maior ou igual ao superior completo & $\begin{array}{l}0,344^{* * *} \\
(0,013)\end{array}$ & $\begin{array}{l}0,305^{* * *} \\
(0,009)\end{array}$ & $\begin{array}{l}0,278^{* * *} \\
(0,010)\end{array}$ \\
\hline Experiência do Homem & $\begin{array}{c}-0,004^{* * *} \\
(0,001)\end{array}$ & $\begin{array}{c}-0,004^{* * *} \\
(0,001)\end{array}$ & $\begin{array}{c}-0,004^{* * *} \\
(0,001)\end{array}$ \\
\hline Escolaridade do homem & $\begin{array}{c}-0,008^{* * *} \\
(0,002)\end{array}$ & $\begin{array}{c}-0,006^{* * *} \\
(0,001)\end{array}$ & $\begin{array}{c}-0,006^{* * *} \\
(0,002)\end{array}$ \\
\hline$D^{-}$ & $\begin{array}{l}0,063^{* * *} \\
(0,017)\end{array}$ & $\begin{array}{l}0,065^{* * *} \\
(0,015)\end{array}$ & $\begin{array}{l}0,036^{* *} \\
(0,016)\end{array}$ \\
\hline$D^{+}$ & $\begin{array}{c}-0,097^{* * *} \\
(0,024)\end{array}$ & $\begin{array}{c}-0,125^{* * *} \\
(0,022)\end{array}$ & $\begin{array}{c}-0,109^{* * *} \\
(0,022)\end{array}$ \\
\hline Interação entre ter filhos entre 0 e 5 anos e $D^{-}$ & $\begin{array}{c}-0,214^{* * *} \\
(0,015)\end{array}$ & $\begin{array}{c}-0,165^{* * *} \\
(0,018)\end{array}$ & $\begin{array}{c}-0,180^{* * *} \\
(0,023)\end{array}$ \\
\hline Interação entre ter filhos entre 0 e 5 anos e Dref & $\begin{array}{c}-0,144^{* * *} \\
(0,014)\end{array}$ & $\begin{array}{c}-0,150^{* * *} \\
(0,013)\end{array}$ & $\begin{array}{c}-0,147^{* * *} \\
(0,013)\end{array}$ \\
\hline Interação entre ter filhos entre 0 e 5 anos e $D^{+}$ & $\begin{array}{c}-0,110^{* * *} \\
(0,040)\end{array}$ & $\begin{array}{c}-0,071^{* *} \\
(0,032)\end{array}$ & $\begin{array}{c}-0,095^{* * *} \\
(0,034)\end{array}$ \\
\hline Interação entre ter filho entre 6 e 10 anos e $D^{-}$ & $\begin{array}{c}-0,038^{* *} \\
(0,015)\end{array}$ & $\begin{array}{c}-0,045^{* * *} \\
(0,017)\end{array}$ & $\begin{array}{c}-0,060^{* * *} \\
(0,021)\end{array}$ \\
\hline Interação entre ter filho entre 6 e 10 anos e Dref & $\begin{array}{c}-0,060^{* * *} \\
(0,014)\end{array}$ & $\begin{array}{r}-0,022^{*} \\
(0,012)\end{array}$ & $\begin{array}{c}-0,048^{* * *} \\
(0,012)\end{array}$ \\
\hline Interação entre ter filhos entre 6 e 10 anos e $D^{+}$ & $\begin{array}{c}0,02 \\
(0,037)\end{array}$ & $\begin{array}{c}-0,007^{* * *} \\
(0,031)\end{array}$ & $\begin{array}{c}0,02 \\
(0,028)\end{array}$ \\
\hline Observações $^{2}$ & 17627 & 18506 & 17394 \\
\hline
\end{tabular}

Observações $^{2}$

As regressões são estimadas usando um modelo Probit. Os valores reportados se referem aos efeitos marginais. Os erros-padrão robustos são reportados entre parênteses. A amostra é composta por mulheres entre 15 e 50 anos que possuem um companheiro homem ${ }^{*}$ siginicativo a $10 \%$; $^{* *}$ significativo a $5 \% ;{ }^{* * *}$ significativo a $1 \%$

Nota 1: $\mathrm{O}$ universo de análise considera apenas as famílias nucleares.

Nota 2: Em todos os modelos foram utilizadas dummies para as regiões metropolitanas. Fonte: PNAD entre 2001 e 2008 do IBGE. 
Em suma, apesar de as mulheres pertencentes a famílias com maior inclinação à pobreza terem maior probabilidade de estar na PEA, esse efeito é anulado, e até revertido, se elas possuírem filhos pequenos. Isso plausivelmente revela tanto uma elevação da sua produtividade familiar nessa situação, assim como a ausência de substitutos para o tempo dedicado aos cuidados dos filhos nessa faixa etária. De fato, nas famílias do grupo $D^{+}$, o impacto da presença de filhos de 0 a 5 anos sobre o ingresso da mulher é bastante suavizado e, no caso de crianças de 6 a 10 anos não chega a ser significativo.

No caso das famílias desse último grupo a menor magnitude do efeito pode ser creditada à capacidade e viabilidade das mulheres, como resultado do cotejo das produtividades em casa e no mercado, de arcar com substitutos para o tempo necessário no cuidado com as crianças, como creches e babás. De modo geral, esses resultados parecem respaldar a conjectura de que o entorno familiar, entendido como o estado de riqueza ou pobreza potencial, influi sobre a decisão de fazer parte da força de trabalho, bem como afeta a importância relativa dos determinantes tradicionais.

\section{Resumo e conclusões}

O estudo buscou contribuir para a investigação acerca da participação feminina no mercado de trabalho e seus determinantes, tanto pelo impacto de características pessoais e domiciliares sobre a decisão de ingresso como, principalmente, por meio da influência do status socioeconômico das famílias. Para tanto foi criado um indicador independente da decisão de ingresso, baseado na escolaridade dos cônjuges e na existência de outras fontes de renda que não o trabalho deles.

A análise empírica no enfoque padrão para o ano de 2008 permitiu ratificar que, no âmbito de famílias nucleares, a experiência e escolaridade do cônjuge masculino, que atuam como proxy de seus rendimentos do trabalho, têm uma influência negativa na participação das mulheres. Da mesma forma, a presença de filhos em idade pré-escolar, com mais intensidade, e de crianças na faixa etária de 6 a 10 anos de idade, são obstáculos de destaques no ingresso feminino na força de trabalho. Por outro lado, maiores níveis de escolaridade mostram um efeito positivo sobre a oferta de trabalho feminina. No que diz respeito à faixa etária, verificou-se que o efeito marginal sobre essa oferta atinge seu maior valor nas mulheres entre 30 e 39 anos.

Nos modelos que consideram a influência do status socioeconômico como indicador de condição potencial de carência da família, foi constatado que a decisão de ingresso das mulheres no mercado de trabalho está associada a essa condição da família. Famílias com menor dotação das características socioeconômicas consideradas - existência de outras fontes de renda e escolaridade dos cônjuges - têm uma probabilidade mais alta de ter a mulher participando da força de trabalho, o que plausivelmente está refletindo o peso relativamente maior dado ao rendimento do trabalho da mulher na formação da renda domiciliar. Já nas famílias mais bem dotadas a probabilidade de ingresso das mulheres diminui.

Por fim, a interação da condição socioeconômica das famílias e a presença de filhos pequenos revelou-se importante. No caso de famílias com menor status socioeconômico que possuem filhos pequenos, o incremento da participação mencionado anteriormente tende a ser atenuado, e mesmo revertidos 
no caso de filhos em idade pré-escolar. Tal efeito deve advir da elevação da produtividade doméstica como resultado da ausência de, ou impossibilidade de arcar com, substitutos para o seu tempo dedicado aos filhos. O fato de esse impacto ser menor, e mesmo inexistente para o caso de crianças entre 6 e 10 anos de idade, respalda essa possibilidade.

Parece correto, então, concluir que o entorno familiar em que a mulher se encontra influi sobre a sua decisão de ingressar no mercado de trabalho e, no caso da presença de filhos menores a redução na taxa de participação da força de trabalho é maior entre as famílias com maior inclinação à pobreza, o que contribuindo para agravar as perspectivas de quem já se encontra em uma condição socioeconômica desvantajosa. Daí a relevância de políticas públicas que ofereçam substitutos para o tempo da mulher dedicado aos filhos menores, como a disponibilidade de creches para crianças em idade pré-escolar, para possibilitar o regate dessas famílias da condição de pobreza.

\section{Referências Bibliográficas}

Barros, R. \& Mendonça, R. (1989), 'Família e distribuição de renda: o impacto da participação das esposas no mercado de trabalho.', Pesquisa e Planejamento Econômico 19.

Barros, R., Ramos, L. \& Santos, E. (1995), Gender differences in brazilian labor markets., in P. Schultz., ed., 'Investment in women's human capital', University of Chicago Press.

Becker, G. (1965), 'A theory of the allocation of time.', Economic Journal 75, 493-517.

Camargo, J. \& Serrano, F. (1983), 'Os dois mercados: homens e mulheres na indústria brasileira.', Rio de Janeiro.

Costa, J. (2007), Determinantes da participação feminina no mercado de trabalho brasileiro., Master's thesis, Universidade de Brasília.

Durand, J. (1975), 'The labor force in economic development: a comparison of international census data 1946-1966', Princeton, Princeton University Press.

Fernandes, R., Pazello, E. \& Felício, F. (2002), 'A importância da estrutura familiar e do engajamento no mercado de trabalho na determinação da pobreza no brasil', Pesquisa e Planejamento Econômico 32.

Hoffmann, R. \& Leone, E. (2004), 'Participação da mulher no mercado de trabalho e desigualdade da renda domiciliar per capita no brasil: 1981-2002', Nova Economia 2.

Melo, M. (1982), Uma análise da participação feminina no mercado de trabalho do brasil., Master's thesis, PUC-RJ.

Ramos, L. \& Soares, A. L. (1995), 'Participação da mulher na força de trabalho e pobreza no brasil.', Rio de Janeiro: Revista de Economia Política 15.

Scorzafave, L. \& Menezes-Filho, N. (2005), 'Impacto da participação das mulheres na evolução da distribuição de renda do trabalho no brasil.', Pesquisa e Planejamento Econômico 35. 
Sedlacek, G. L. \& Santos, E. C. (1991), 'A mulher cônjuge no mercado de trabalho como estratégia de geração da renda familiar.', Pesquisa e Planejamento Econômico 21.

\section{Apêndice A}

Tabela A.1: Distribuição do indicador D, segundo os décimos da distribuição da renda familiar per capita de 2008

\begin{tabular}{lrrrrrrrrrr}
\hline Décimo & \multicolumn{1}{c}{1} & \multicolumn{1}{c}{2} & \multicolumn{1}{c}{3} & \multicolumn{1}{c}{4} & \multicolumn{1}{c}{5} & \multicolumn{1}{c}{6} & \multicolumn{1}{c}{7} & \multicolumn{1}{c}{8} & \multicolumn{1}{c}{9} & 10 \\
\hline$D^{-}$ & 14,6 & 15,7 & 15,1 & 14,1 & 12,6 & 11,0 & 8,7 & 5,5 & 2,2 & 0,5 \\
$D^{0}$ & 7,1 & 8,5 & 8,8 & 9,1 & 9,9 & 10,4 & 10,9 & 11,5 & 12,2 & 11,7 \\
$D^{+}$ & 6,5 & 7,6 & 8,4 & 8,3 & 7,0 & 7,6 & 8,8 & 11,4 & 13,6 & 20,8 \\
\hline
\end{tabular}


Tabela A.2: Estatísticas descritivas para o Brasil metropolitano - 2001, 2005 e 2008

\begin{tabular}{|c|c|c|c|}
\hline Variáveis & 2001 & 2005 & 2008 \\
\hline \multicolumn{4}{|l|}{ Características das Mulheres } \\
\hline Mulheres com filhos (\%) & 85,8 & 84,3 & 81,6 \\
\hline Mulheres sem filhos (\%) & 14,2 & 15,7 & 18,4 \\
\hline Número médio de filhos & 1,8 & 1,7 & 1,6 \\
\hline Entre 0 e 5 anos & 0,5 & 0,4 & 0,4 \\
\hline Entre 6 e 10 anos & 0,4 & 0,4 & 0,4 \\
\hline Entre 11 e 17 anos & 0,6 & 0,5 & 0,5 \\
\hline Com 18 anos ou mais & 0,3 & 0,3 & 0,3 \\
\hline Idade Média & 34,7 & 35,3 & 35,8 \\
\hline Entre 15 e 19 anos (\%) & 2,5 & 2,0 & 1,6 \\
\hline Entre 20 e 29 anos (\%) & 27,7 & 25,6 & 23,6 \\
\hline Entre 30 e 39 anos (\%) & 38,2 & 38,3 & 38,7 \\
\hline Entre 40 e 50 anos (\%) & 31,7 & 34,1 & 36,1 \\
\hline Educação Média & 7,8 & 8,5 & 9,2 \\
\hline $\begin{array}{l}\text { Com no máximo o ensino fundamental incompleto } \\
(\%)\end{array}$ & 44,1 & 36,7 & 29,4 \\
\hline $\begin{array}{l}\text { Entre o ensino fundamental completo e o médio in- } \\
\text { completo }(\%)\end{array}$ & 21,3 & 20,1 & 20,0 \\
\hline $\begin{array}{l}\text { Entre o ensino médio completo e o superior incom- } \\
\text { pleto }(\%)\end{array}$ & 25,9 & 32,6 & 37,0 \\
\hline Com no mínimo o superior completo (\%) & 8,8 & 10,6 & 13,6 \\
\hline Ocupadas (\%) & 50,2 & 56,5 & 60,5 \\
\hline Desocupadas (\%) & 7,8 & 9,8 & 7,1 \\
\hline \multicolumn{4}{|l|}{ Características dos Homens } \\
\hline Idade Média & 38,5 & 39,0 & 39,4 \\
\hline Educação Média & 7,7 & 8,4 & 8,9 \\
\hline Ocupados (\%) & 88,4 & 90,3 & 91,8 \\
\hline Desocupados (\%) & 5,5 & 4,5 & 3,0 \\
\hline Características do status sócio-econômico do domicílio & & & \\
\hline$D^{+}$ou potencialmente ricos $(\%)$ & 7,5 & 9,2 & 10,1 \\
\hline Dref ou grupo de referência (\%) & 51,6 & 61,4 & 68,3 \\
\hline$D^{-}$ou potencialmente pobres $(\%)$ & 41,0 & 29,5 & 21,6 \\
\hline
\end{tabular}

Fonte: Elaboração própria a partir da PNAD de 2001, 2005 e 2008 


\section{Apêndice B}

Tabela B.1: Participação da cônjuge mulher na força de trabalho entre 2001, 2005 e 2008 - Coeficientes

\begin{tabular}{|c|c|c|c|}
\hline & \multicolumn{3}{|c|}{ Ano } \\
\hline & 2001 & 2005 & 2008 \\
\hline Tem filhos de 11 a 17 anos & $\begin{array}{l}0,043 \\
(0,028)\end{array}$ & $\begin{array}{l}0,062^{* *} \\
(0,027)\end{array}$ & $\begin{array}{l}0,034 \\
(0,028)\end{array}$ \\
\hline Cônjuge entre 15 e 19 anos & & & \\
\hline Cônjuge entre 20 e 29 anos & $\begin{array}{l}0,525^{* * *} \\
(0,076)\end{array}$ & $\begin{array}{l}0,423^{* * *} \\
(0,081)\end{array}$ & $\begin{array}{l}0,603^{* * *} \\
(0,092)\end{array}$ \\
\hline Cônjuge entre 30 e 39 anos & $\begin{array}{l}0,665^{* * *} \\
(0,080)\end{array}$ & $\begin{array}{l}0,484^{* * *} \\
(0,084)\end{array}$ & $\begin{array}{l}0,646^{* * *} \\
(0,095)\end{array}$ \\
\hline Cônjuge entre 40 e 50 anos & $\begin{array}{l}0,446^{* * *} \\
(0,086)\end{array}$ & $\begin{array}{l}0,321^{* * *} \\
(0,088)\end{array}$ & $\begin{array}{l}0,506^{* * *} \\
(0,099)\end{array}$ \\
\hline Escolaridade entre 0 e 7 anos & - & - & - \\
\hline Escolaridade entre 8 e 11 anos & $\begin{array}{l}0,122^{* * *} \\
(0,033)\end{array}$ & $\begin{array}{l}0,212^{* * *} \\
(0,033)\end{array}$ & $\begin{array}{l}0,070^{* *} \\
(0,035)\end{array}$ \\
\hline Escolaridade entre 11 anos e superior incompleto & $\begin{array}{l}0,498^{* * *} \\
(0,039)\end{array}$ & $\begin{array}{l}0,494^{* * *} \\
(0,036)\end{array}$ & $\begin{array}{l}0,370^{* * *} \\
(0,036)\end{array}$ \\
\hline Escolaridade maior ou igual ao superior completo & $\begin{array}{l}1,121^{* * *} \\
(0,062)\end{array}$ & $\begin{array}{l}1,181^{* * *} \\
(0,060)\end{array}$ & $\begin{array}{l}1,028^{* * *} \\
(0,057)\end{array}$ \\
\hline Experiência do Homem & $\begin{array}{c}-0,011^{* * *} \\
(0,002)\end{array}$ & $\begin{array}{c}-0,011^{* * *} \\
(0,002)\end{array}$ & $\begin{array}{c}-0,012^{* * *} \\
(0,002)\end{array}$ \\
\hline Escolaridade do homem & $\begin{array}{c}-0,020^{* * *} \\
(0,004)\end{array}$ & $\begin{array}{c}-0,017^{* * *} \\
(0,004)\end{array}$ & $\begin{array}{c}-0,016^{* * *} \\
(0,004)\end{array}$ \\
\hline$D^{-}$ & $\begin{array}{l}0,161^{* * *} \\
(0,043)\end{array}$ & $\begin{array}{l}0,185^{* * *} \\
(0,044)\end{array}$ & $\begin{array}{l}0,103^{* *} \\
(0,047)\end{array}$ \\
\hline$D^{+}$ & $\begin{array}{c}-0,245^{* * *} \\
(0,061)\end{array}$ & $\begin{array}{c}-0,331^{* * *} \\
(0,055)\end{array}$ & $\begin{array}{c}-0,293^{* * *} \\
(0,056)\end{array}$ \\
\hline Interação entre ter filhos entre 0 e 5 anos e $D^{-}$ & $\begin{array}{c}-0,544^{* * *} \\
(0,039)\end{array}$ & $\begin{array}{c}-0,433^{* * *} \\
(0,046)\end{array}$ & $\begin{array}{c}-0,474^{* * *} \\
(0,057)\end{array}$ \\
\hline Interação entre ter filhos entre 0 e 5 anos e Dref & $\begin{array}{c}-0,366^{* * *} \\
(0,036)\end{array}$ & $\begin{array}{c}-0,401^{* * *} \\
(0,033)\end{array}$ & $\begin{array}{c}-0,398^{* * *} \\
(0,034)\end{array}$ \\
\hline Interação entre ter filhos entre 0 e 5 anos e $D^{+}$ & $\begin{array}{c}-0,277^{* * *} \\
(0,099)\end{array}$ & $\begin{array}{c}-0,190^{* *} \\
(0,083)\end{array}$ & $\begin{array}{c}-0,254^{* * *} \\
(0,088)\end{array}$ \\
\hline Interação entre ter filho entre 6 e 10 anos e $D^{-}$ & $\begin{array}{c}-0,096^{* * *} \\
(0,037)\end{array}$ & $\begin{array}{c}-0,123^{* * *} \\
(0,045)\end{array}$ & $\begin{array}{c}-0,165^{* * *} \\
(0,055)\end{array}$ \\
\hline Interação entre ter filho entre 6 e 10 anos e Dref & $\begin{array}{c}-0,152^{* * *} \\
(0,036)\end{array}$ & $\begin{array}{c}-0,061^{*} \\
(0,033)\end{array}$ & $\begin{array}{c}-0,132^{* * *} \\
(0,033)\end{array}$ \\
\hline Interação entre ter filhos entre 6 e 10 anos e $D^{+}$ & $\begin{array}{l}0,53 \\
(0,097)\end{array}$ & $\begin{array}{c}-0,02 \\
(0,085)\end{array}$ & $\begin{array}{l}0,058 \\
(0,080)\end{array}$ \\
\hline Observações $^{2}$ & 17627 & 18506 & 17394 \\
\hline
\end{tabular}

As regressões são estimadas usando um modelo Probit. Os valores reportados se referem aos efeitos marginais. Os erros-padrão robustos são reportados entre parênteses. A amostra é composta por * siginicativo a $10 \%$; ${ }^{* *}$ significativo a $5 \%$; $* * *$ significativo a $1 \%$

Nota 1: O universo de análise considera apenas as famílias nucleares.

Nota 2: Em todos os modelos foram utilizadas dummies para as regiões metropolitanas.

Fonte: PNAD entre 2001 e 2008 do IBGE. 